\title{
Realistic many-body approaches to materials with strong nonlocal correlations
}

\author{
F. Lechermann ${ }^{1,2}$, A.I. Lichtenstein ${ }^{1}$ and M. Potthoff ${ }^{1, a}$ \\ 1 I. Institut für Theoretische Physik, Universität Hamburg, Jungiusstraße 9, \\ 20355 Hamburg, Germany \\ 2 Institut für Keramische Hochleistungswerkstoffe, Technische Universität \\ Hamburg-Harburg, 21073 Hamburg, Germany
}

Received 13 February 2017 / Received in final form 10 March 2017 Published online 10 July 2017

\begin{abstract}
Many of the fascinating and unconventional properties of several transition-metal compounds with partially filled $d$-shells are due to strong electronic correlations. While local correlations are in principle treated exactly within the frame of the dynamical mean-field theory, there are two major and interlinked routes for important further methodical advances: On the one hand, there is a strong need for methods being able to describe material-specific aspects, i.e., methods combining the DMFT with modern band-structure theory, and, on the other hand, nonlocal correlations beyond the mean-field paradigm must be accounted for. Referring to several concrete example systems, we argue why these two routes are worth pursuing and how they can be combined, we describe several related methodical developments and present respective results, and we discuss possible ways to overcome remaining obstacles.
\end{abstract}

\section{Introduction}

The quantum theory of the electronic structure of solids is very much characterized by the dichotomy of spatially local and nonlocal concepts. Nonlocality first comes across when considering the Hamiltonian of an electron moving in a potential $V$ with the discrete translational symmetries of an infinite three-dimensional lattice: Its eigenstates are highly nonlocal and extend over the entire system, and the spectrum of eigenenergies, the band structure, lives in reciprocal or $\mathbf{k}$-space. While this band-structure problem cannot be solved analytically, there are nowadays extremely efficient and highly reliable computational tools to solve the single-electron Schrödinger equation for rather general lattice potentials [1-4].

Thanks to density-functional theory (DFT) [5-7], the solution of the band problem is key to a quantitative theory with predictive power: According to this paradigm of electronic-structure theory established in the 1960s, essentially independent electrons move in an effective one-particle lattice potential $V$ which, on a static mean-field level, includes effects of Fermi statistics ("exchange") and of "correlations". The success of band theory is very much due to the fact that $V=V(\mathbf{r})$ is local - opposed to the difficult-to-handle nonlocal exchange term of Hartree-Fock theory, for example.

\footnotetext{
${ }^{a}$ e-mail: michael.potthoff@physik.uni-hamburg.de
} 
Essential for a realistic modeling of the electronic structure is the treatment of electronic correlations. This central notion refers to all physics beyond the concept of a single Slater determinant and beyond a simple construction of the ground state by filling electrons, according to the Aufbau principle, into the nonlocal one-particle eigenstates up to the Fermi energy. Opposed to configuration-interaction schemes for atomic or molecular systems, an explicit treatment of correlations by means of the full $N$-electron wave function is not practicable since for solids one has to consider a macroscopically large number of electrons. It is thus advisable to shift the main focus to a different fundamental quantity of interest: Most suitable in the context of the Hohenberg-Kohn theory is the local electron density $n(\mathbf{r})$. This allows for an average and static mean-field-like description of correlation effects by means of the local-density approximation (LDA) and extensions [6,7].

In the recent decades, however, more and more material classes have been discovered, have been designed or simply have moved in the focus of materials science, where the DFT-LDA approach breaks down. In most cases this is related to correlation phenomena like spontaneous magnetic order [8], correlation-driven metal-insulator transitions [9] or high-temperature superconductivity [10]. This comprises $d$-electron materials and $3 d$ transition-metal compounds, for instance. Typical examples are oxides, such as the intensively discussed unconventional cuprate-based superconductors, or pnictides, in particular iron-pnictide superconductors, cobaltates, etc. but also Mott or charge-transfer insulators like transition-metal monoxides, or simply late $3 d$ transition metals as the classical band ferromagnets Fe, Co, Ni. These "strongly correlated electron systems" require a more explicit treatment of correlations which is conventionally done with the help of Feynman diagrams, i.e., in the context of many-body perturbation theory [11]. The central physical quantity here is the single-electron propagator or Green's function $G\left(\mathbf{r}, \mathbf{r}^{\prime}, t, t^{\prime}\right)$ which is spatially nonlocal. The Green's function is closely related to photoemission spectroscopy [12] and describes the propagation of the additional hole in the occupied part of the band structure that is left in the final state; it thus describes a nonlocal process. Note that temporal homogeneity implies that $G$ depends on the time difference only and, via Fourier transformation, can be written as a function of the excitation energy $\omega$.

In the 1990s an important discovery was made for many-electron models with local interactions, such as the famous Hubbard model [13-15]: The summation of all local (renormalized skeleton) diagrams contributing to the electronic self-energy can be achieved in practice by solving an effective impurity problem, the parameters of which must be determined self-consistently. This idea constitutes the dynamical mean-field theory (DMFT) [16-19], a powerful nonperturbative and internally consistent mean-field theory which exactly accounts for the local quantum fluctuations while nonlocal correlations are treated on average only. A practical DMFT calculation must be based on an "impurity solver". There are different highly sophisticated variants available, based on Krylov-space techniques, quantum Monte-Carlo simulations or renormalization-group techniques, for example [20-24]. The success of DMFT is largely due to the fact that the importance of nonlocal correlations diminishes with increasing spatial dimension and that this effect is already relevant for three dimensions. In fact, the DMFT is the exact theory of the Hubbard model on an infinite-dimensional lattice. DMFT is also a versatile theory which is easily adapted to different lattice-fermion models and which has been extended in various directions.

Nonlocal correlation effects, i.e., correlations beyond the DMFT, are key to understanding and controlling the electronic properties of several important material classes. Still, the dynamical mean-field theory can serve as a starting point. The purpose of the present work is to discuss electronic-structure theory and materials science from this perspective. Referring to a couple of concrete and typical examples, 
we review some essential theoretical aspects and recent methodical advances in this field.

Section 2 provides an introduction to the issue of local and nonlocal effects and what can be described by means of the DMFT. As an instructive example we discuss the subtle competition between the Kondo effect and the indirect magnetic exchange in a periodic Anderson model on the triangular lattice. Even on the pure model level, however, there are important effects of nonlocal correlations beyond DMFT, in particular the feedback of nonlocal two-particle correlations on the electronic selfenergy. Several proposals have been made to access nonlocal effects using DMFT as a platform for further improvements. Besides cluster extensions [25] or further diagram resummations [26], this comprises the idea of dual-fermion theory [27], reviewed in Section 3, and also includes the extension to the dual-boson technique [28], which is in addition capable of handling nonlocal interactions.

The combination of DMFT with band theory is a fascinating vision with the final goal of establishing a parameter-free computational approach, covering largely different material classes. This ongoing development, described in Section 4, is highly ambitious as it requires to unify nonlocal concepts from band theory with the locality paradigm of DMFT. The discussion of $\mathrm{V}_{2} \mathrm{O}_{3}$ as a concrete example sheds some light on the current state of the art. Novel materials-design ideas are touched upon by discussing application to the prominent problem of oxide heterostructures. In view of realistic nonlocal self-energy effects, we discuss in Section 5 spin-polaron physics in $\mathrm{Na}_{x} \mathrm{CoO}_{2}$, based on applied dual-fermion theory.

\section{Local vs. nonlocal correlations}

Let us start our discussion with an application of the DMFT to a "simple" model system. Without going too much into the formalism and by concentrating on the physics, this should help to qualitatively understand the capabilities of the DMFT but also show what is beyond a dynamic mean-field approach.

One of the hallmarks of strong correlations among itinerant valence electrons is the formation of local magnetic moments [29]. Local-moment formation is constitutive for much of the physics captured by the periodic Anderson model (PAM) which is the most simple model for heavy-fermion materials [30,31]. Generically, the PAM describes a band of light conduction electrons (" $c$ electrons") of bandwidth $W$ hybridizing, with local hybridization strength $V$, with a narrow band of " $f$ electrons" located at energy $\varepsilon_{f}$. Since the $f$ band is narrow, Coulomb correlations are important and are taken into account by an on-site Hubbard interaction $U$.

In the local-moment regime of the PAM for strong $U$, charge fluctuations on the local $f$ orbitals are effectively suppressed. The single-electron spectrum exhibits two Hubbard bands located around $\varepsilon_{f}$ and $\varepsilon_{f}+U$ which are well separated from the $c$ band located around the chemical potential $\mu$, i.e., $\varepsilon_{f} \ll \mu \ll \varepsilon_{f}+U$, and each $f$ orbital is exactly occupied by one electron. In this limit, the low-energy physics is perturbatively captured by the more simple Kondo-lattice model [31,32], where the $f$ degrees of freedom are represented by quantum spins with $S_{f}=1 / 2$. Mediated by a super-exchange mechanism [33], the localized magnetic moments on the $f$ orbitals couple to the $c$-electron spins via a local antiferromagnetic exchange of strength $J=8 V^{2} / U$. This coupling $J$ lifts the macroscopic ground-state spin degeneracy and quenches the residual entropy.

There are however, two different and competing mechanisms, RKKY coupling and Kondo screening, which generate the famous Doniach diagram [34] and corresponding quantum phase transitions [35]. The nonlocal Ruderman-Kittel-Kasuya-Yosida (RKKY) [36] indirect magnetic exchange is mediated via magnetic polarization of 


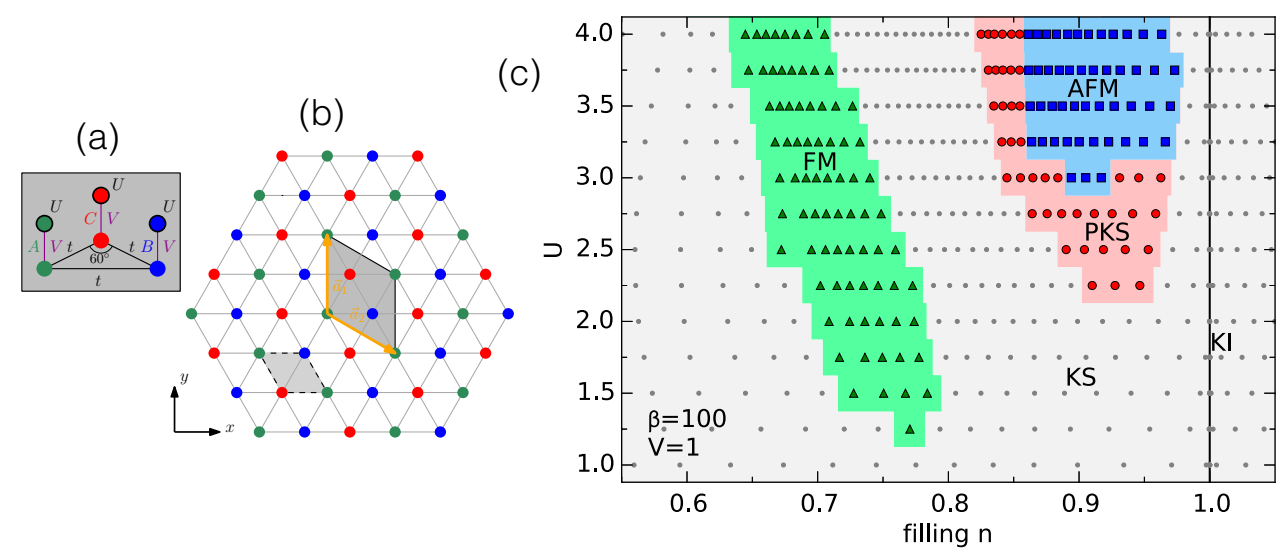

Fig. 1. Adapted from reference [39]: Magnetic phase diagram of the periodic Anderson model on the triangular lattice as obtained by site-dependent DMFT. (a) Unit cell used for the calculations. Sites A, B, C are treated independently. For each site, an $f$ orbital with local interaction $U$ couples to a $c$ orbital via the hybridization $V$. The nearest-neighbor hopping $t=1$ sets the energy scale. (b) Triangular lattice with primitive cell (light gray, dashed lines) and the unit cell used for the calculations (gray, solid lines). (c) Phase diagram in the plane spanned by $U$ and the filling $n$ (total electron number per site) at inverse temperature $\beta=100$. KI: Kondo insulator (at half-filling $n=1$ ), KS: metallic Kondo singlet state, PKS: partial Kondo-singlet phase, AFM: antiferromagnetic phase, FM: ferromagnetic phase.

the conduction electrons; it dominates for weak $J$, and is characterized by the effective coupling strength $J_{\mathrm{RKKY}}(\mathbf{q})=-J^{2} \chi_{c}(\mathbf{q}, \omega=0)$ where $\chi_{c}$ is the $c$-electron spin susceptibility. The Kondo screening of the $f$ magnetic moments is a local correlation effect which is already captured in the impurity variants of the Kondo or Anderson model and dominates at stronger $J$. Below the characteristic Kondo scale [37,38] given by $T_{\mathrm{K}} \propto e^{-W / J}$, the $f$ moment forms a singlet with a mesoscopically extended cloud of $c$-electron moments.

Figure 1c shows the phase diagram as obtained [39] by DMFT using the continuous-time quantum Monte-Carlo method (CT-QMC) [22,40] based on the hybridization expansion and the segment algorithm [41] at a low temperature $(\beta=$ $\left.1 / k_{\mathrm{B}} T=100\right)$. Each point corresponds to a converged DMFT calculation. At halffilling $n=1$, the hybridization band gap in the noninteracting density of states results in a band insulator for $U=0$. This develops into a correlated Kondo insulator (KI) with increasing $U$. Furthermore, we find a metallic ferromagnetic phase (FM) at low fillings in the mixed-valence regime where the $f$ local moments are no longer well defined. Most interesting, however, is the competition between an antiferromagnetic phase (AFM) and the Kondo-singlet state (KS) in the local-moment regime for fillings slightly off half-filling. As expected, the RKKY-induced AFM phase shows up for strong $U$, i.e., weak $J$, while the metallic heavy-fermion KS state, which connects to the KI at $n=1$, is realized at weaker $U$ but prior to charge fluctuations becoming dominant.

The competition between Kondo screening and RKKY coupling is affected by the triangular lattice geometry (see Fig. 1b). The nonbipartiteness of the lattice already explains the asymmetry of the phase diagram under particle-hole transformations: Choosing a positive hopping $t=1$ implies that the center of gravity of the noninteracting total density of states is located close to the lower band edge and that symmetry-broken magnetic phases are expected for fillings below half-filling. 
More importantly, however, the lattice also introduces frustration of the antiferromagnetic ordering tendency, and thus the problem gets more involved due to another energy scale which is associated with the release of frustration. For the PAM or the Kondo lattice, a mechanism of partial Kondo screening (PKS) [42] has been suggested where a spontaneous, site-selective Kondo effect (say, on site A in the unit cell, see Fig. 1a) alleviates the frustration and thus allows the remnant spins (on sites B and C) to order magnetically via the RKKY coupling. This is an interesting compromise between a local and a nonlocal correlation effect which has attracted considerable attention in the past $[43,44]$ and which has been studied in the PAM on the level of the static mean-field (Hartree-Fock) approximation $[45,46]$. The dynamical meanfield study discussed here correctly includes all local fluctuations and also captures the Kondo effect. Apparently this is sufficient to destroy any magnetic order at $n=1$ found in the static theory [45].

The phase diagram is actually obtained by applying a variant of DMFT ("sitedependent DMFT") where the different correlated orbitals in the unit cell are treated independently, similar to a real-space DMFT approach [47]. In fact, a spontaneous breaking of the $120^{\circ}$ rotational symmetry is found at the border between the AFM and KS phase (Fig. 1c). At this border it becomes favorable to avoid frustration by partial Kondo screening of one out of three $f$ moments. A detailed study [39] shows that the PKS state is metallic and that it supports an additional weak chargedensity-wave ordering, mainly on the $c$ orbitals. Furthermore, due to proximity to the RKKY-coupled remnant moments, the corresponding breaking of time-reversal symmetry results in a slightly imperfect partial Kondo screening with a tiny residual magnetic moment on the $f$ orbital at the Kondo site in the unit cell. DMFT predicts a robust PKS in a large parameter range. Anisotropies [42] are not necessary to stabilize the phase. PKS appears at noninteger fillings, i.e., the gain in kinetic energy might be essential to stabilize the phase such that spin-only models would be ruled out. Steering the system through the border between the paramagnetic heavy-fermion and the magnetically ordered phase could be an experimental route to detect the PKS phase.

There are some lessons learned about the dichotomy between local and nonlocal concepts in electronic-structure theory and about the point where DMFT stands: The Kondo effect is due to a highly nonlocal singlet formation but is fully included in the framework of DMFT since it is driven by a local (exchange) interaction between a local magnetic moment and an uncorrelated bath. The metallic Kondo singlet (KS) or heavy-fermion state of the PAM, on the other hand, is much more intricate as it must build from coherently overlapping Kondo singlets. DMFT can only provide an approximation to the physics on this coherence scale at energies even lower than the impurity Kondo scale. This influences the competion with the RKKY coupling in an essentially unknown way. The RKKY interaction itself is a nonlocal interaction and thus cannot be treated explicitly with the standard single-site DMFT. In the PAM, however, it is generated perturbatively as an effective interaction. Hence, DMFT does capture its full spatial structure and therewith the corresponding tendencies towards magnetic ordering. However, DMFT does not include the feedback of nonlocal magnetic correlations on the one-particle self-energy, induced by the RKKY coupling, as only local diagrams are summed up. Those missing fluctuations must result in mean-field artifacts. Typically, the DMFT will be biased, to some extent, toward magnetic ordering and a spontaneously symmetry-broken state $|\uparrow\rangle|\downarrow\rangle$ at the expense of a nonlocal singlet $(|\uparrow\rangle|\downarrow\rangle-|\downarrow\rangle|\uparrow\rangle) / \sqrt{2}[48,49]$. Although one could thus argue that the AFM state is overestimated in the phase diagram, compared to the Kondo singlet state, one would still expect a PKS phase at the border between both phases. In any case, given the complexity of a problem posed by strong correlations in fermionic models on two-dimensional frustrated lattices with several mechanisms competing 
on low energy scales, the DMFT cannot be expected to provide more than a useful starting point. Cluster and other extensions of the single-site mean-field concept, see below, can be invoked to progressively include the effects of short- and long-range correlations.

\section{Nonlocal correlations}

There are several strategies to include nonlocal correlations beyond the DMFT which can be explained by referring to the the Hubbard model. Here, we describe the dualfermion [27] and the dual-boson approach [28] but start with a short discussion of DMFT again.

Consider the noninteracting, "kinetic" part $H_{0}$ of the Hubbard model first. This is fixed by specifying the hopping-matrix elements $t_{i j}$ between sites $i$ and $j$. In the absence of the local Hubbard-interaction term, $H_{0}$ is easily diagonalized. For a Hubbard model on a translationally invariant lattice with periodic boundary conditions, diagonalization is achieved by Fourier transformation to k-space, and the one-particle excitations are fully captured by the "band structure" $\varepsilon(\mathbf{k})$ with band width $W$. If, on the other hand, only the local part of the Hamiltonian is kept, i.e., the Hubbard interaction $H_{1}$ with interaction strength $U$ and the local term of $H_{0}$ fixed by the on-site energy $\varepsilon_{0}$, the diagonalization of the Hamiltonian is trivial again and essentially reduces in real space to the diagonalization of a single "Hubbard atom".

The great success of the DMFT is related to its ability to interpolate between these two extreme limits, or, at half-filling in particular, between the weak-coupling $(U / W \ll 1)$ metallic state and the strong-coupling $(U / W \gg 1)$ Mott-insulating paramagnetic state [18] in a nonperturbative way which treats the local correlations exactly. On the operational level, DMFT replaces the correlated lattice problem (the Hubbard model) by an impurity problem with a single interacting site and with the same local Hubbard interaction strength $U$, typically by an Anderson impurity model. The one-particle parameters of this impurity model, which are fully determined by the local hybridization function $\Delta(\omega)$, must be determined self-consistently,

$$
\Delta(\omega)=\omega-\varepsilon_{0}-\Sigma(\omega)-\frac{1}{G_{\mathrm{loc}}(\omega)},
$$

from the (local) self-energy $\Sigma$ and the local element of the one-particle Green's function $G_{\text {loc }}$ of the lattice model. The impurity problem, defined in this way, must be solved numerically to get the Green's function on the impurity $G_{\text {imp }}$ and the local impurity self-energy. The self-consistency loop is closed by identifying the impurity self-energy with the lattice-model self-energy, assumed as local, and by calculating the lattice Green's function using Dyson's equation of the lattice model.

A rather obvious idea suggests itself to incorporate nonlocal correlations beyond the DMFT: We start from a self-consistent DMFT solution defined by a self-consistent hybridization function $\Delta(\omega)$ of the Anderson impurity model. Since the Hubbard and the Anderson-impurity model share the same interaction part, one can think of the Hubbard model as the impurity model plus a residual term $\propto \varepsilon(\mathbf{k})-\Delta(\omega)$ and treat this perturbatively. This requires a novel perturbation theory. One may view this idea as a generalization of the Kohn-Sham idea in DFT of an optimal reference system, but with a crucial difference. Here, not an interacting homogeneous electron gas, but an effective impurity model, tailored to the problem of strong correlations, serves as the reference system, see Figure 2.

Since at the zeroth order of this perturbative expansion, i.e., on the level of the DMFT problem, we already have an interacting problem and since the perturbation 


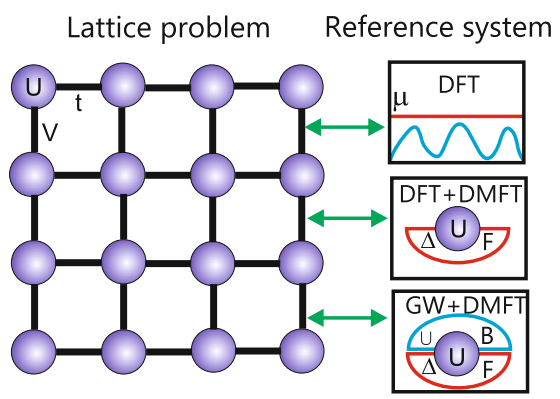

Fig. 2. Sketch of three different approaches to describe many-body effects in lattice-fermion models: (i) Density-functional theory (DFT) with the interacting homogeneous electron gas as a reference system, defined by a constant external potential $\mu$. (ii) Dynamical mean-field theory (DMFT) with an effective impurity problem as a reference system, i.e., a representative correlated atom embedded into a fermionic bath, specified by hybridization function $\Delta$. (iii) GW + DMFT with a more complex impurity reference system, consisting of a correlated atom in a fermionic $(\Delta)$ and a bosonic bath $(\Lambda)$. The latter describes effects of the frequency-dependent screening of long-range Coulomb $(V)$ interactions.

is momentum and frequency dependent, one is forced to replace the Hamiltonians by actions within the path-integral formalism. Note that the fermion path integral can also be used to formulate the DMFT itself $[18,50]$. Now, the separation of the local and nonlocal terms is achieved by a Hubbard-Stratanovich transformation applied to the single-particle $(\varepsilon-\Delta)$-term. This provides us with a new action. Moreover, it is formally possible to integrate out the original local degrees of freedom and in this way generated an effective action in the transformed, so-called dual-fermion representation [27]. Note that integrating out the local degrees of freedom is not only a formal step but can be achieved in practice, namely by numerically solving the problem given by the local impurity action with the help of the CT-QMC method.

The dual action consists of a bare dual propagator $\tilde{G}_{0}(\mathbf{k}, \omega)=\left[G_{\text {imp }}^{-1}(\omega)+\Delta(\omega)-\right.$ $\varepsilon(\mathbf{k})]^{-1}-G_{\text {imp }}(\omega)$, and a local but frequency-dependent effective potential related to scattering processes of two, three, and more dual particles on the impurity site. The simplest two-particle dual potential coincides with the fully connected part of the impurity vertex $\Gamma_{\mathrm{imp}}^{\nu \omega \omega^{\prime}}$, which can be calculated with the impurity CT-QMC solver as a function of bosonic $(\nu)$ and fermionic $\left(\omega, \omega^{\prime}\right)$ Matsubara frequencies. Normally, correlations between three particles on the DMFT impurity site are much weaker than two-particle correlations and can be ignored. The same applies to higher-order terms. Formalizing this argument, one can think of the dual-fermion formalism as an expansion in the order of local multi-particle correlation functions. This means that interactions between dual fermions are related with the connected part of the impurity vertex. Standard diagrammatic techniques can be applied for calculations of the full dual propagator $\tilde{G}_{0}(\mathbf{k}, \omega)$, which allows to obtain the nonlocal self-energy for the original fermions [27] and to describe nonlocal correlations starting from the DMFT solution.

The dual-fermion approach is not necessarily bound to a specific starting point. However, the DMFT starting point is very efficient. Namely, it corresponds to the elimination of all local diagrams for any $n$-particle correlation of dual fermions when using the DMFT self-consistency equation (1). In the dual space, this simply reduces to $\sum_{\mathbf{k}} \tilde{G}_{0}(\mathbf{k}, \omega)=0$ and means that, on average over the whole Brillouin zone, $\Delta(\omega)$ optimally approximates the electron spectrum $\varepsilon(\mathbf{k})$, including its local correlation effects. Therefore, the noninteracting dual fermions correspond to strongly correlated DMFT quasiparticles, and the remaining nonlocal effects can be quite small and 
reasonably described by, e.g., ladder summations of dual diagrams. This also explains the notion "dual fermions".

More interesting but also more complicated many-body nonlocal screening effects can appear if the original Hamiltonian contains additional nonlocal interactions $V(\mathbf{q})$. In this case, one can formulate a similar so-called dual-boson theory [28], which not only takes into account nonlocal fermionic propagators but also bosonic ones, which are screened by the long-range Coulomb interaction. In this way, one can describe, e.g., plasmons or magnons in strongly correlated materials. The action of this "extended" Hubbard model reads as

$$
S=-\sum_{\mathbf{k} \nu \sigma}\left[i \omega+\mu-\varepsilon_{\mathbf{k}}\right] c_{\mathbf{k} \omega \sigma}^{+} c_{\mathbf{k} \omega \sigma}+\frac{1}{2} \sum_{\mathbf{q} \nu} U_{\mathbf{q}} \rho_{\mathbf{q} \nu}^{*} \rho_{\mathbf{q} \nu},
$$

where the Grassmann variables $c_{\mathbf{k} \omega \sigma}^{+}\left(c_{\mathbf{k} \omega \sigma}\right)$ correspond to creation (annihilation) of an electron with momentum $\mathbf{k}$, spin projection $\sigma$, and fermionic Matsubara frequency $\omega$. The interaction $U_{\mathbf{q}}=U+V_{\mathbf{q}}$ consists of the on-site (Hubbard) term and the nonlocal long-range Coulomb interaction, respectively. The screened Coulomb interaction can be frequency dependent as in the case of the constrained random-phase approximation (c-RPA), thus not producing any problems for the present formalism. The charge fluctuations are described by the complex bosonic variable $\rho_{\nu}=n_{\nu}-\langle n\rangle \delta_{\nu}$, where $n_{\nu}=\sum_{\omega \sigma} c_{\omega}^{+} c_{\nu+\omega}$ counts the number of electrons, and $\nu$ is a bosonic Matsubara frequency. For simplicity, we do not include spin degrees of freedom, which could be done by introducing vector spin-boson variables [28]. Moreover we will consider only a one-band model, but keep the matrix form of all equations for a generalization to multi-orbital cases.

As discussed above, we split the lattice action, equation (2), into a sum of effective single-site impurity reference actions $S_{\mathrm{imp}}$, defined by the hybridization function $\Delta_{\omega}$ and by the screened local interaction $\mathcal{U}_{\nu}$, and into a remaining nonlocal part $\tilde{S}=$ $\sum_{i} S_{\mathrm{imp}}^{(i)}+\Delta S$. The contributions to the latter are given by

$$
\begin{aligned}
S_{\mathrm{imp}} & =-\sum_{\omega \sigma}\left[i \omega+\mu-\Delta_{\omega}\right] c_{\omega \sigma}^{+} c_{\omega \sigma}+\frac{1}{2} \sum_{\nu} \mathcal{U}_{\nu} \rho_{\nu}^{*} \rho_{\nu}, \\
\Delta S & =\sum_{\mathbf{k} \omega \sigma} \tilde{\varepsilon}_{\mathbf{k} \omega} c_{\mathbf{k} \omega \sigma}^{+} c_{\mathbf{k} \omega \sigma}+\frac{1}{2} \sum_{\mathbf{q} \nu} \tilde{U}_{\mathbf{q} \nu} \rho_{\mathbf{q} \nu}^{*} \rho_{\mathbf{q} \nu} .
\end{aligned}
$$

Here, $\tilde{\varepsilon}_{\mathbf{k} \omega}=\varepsilon_{\mathbf{k}}-\Delta_{\omega}$ and $\tilde{U}_{\mathbf{q} \nu}=U_{\mathbf{q}}-\mathcal{U}_{\nu}$. One can see that it is easy to incorporate the frequency dependence of the bare Coulomb interaction. The strategy here is similar to the dual-fermion scheme and consists of an efficient perturbation scheme for $\Delta S$ in the action formalism. However, in addition to a fermionic Hubbard-Stratonovich transformation of the first term in equation (3), we also need to perform a standard bosonic transformation of the second (interaction) term. Following the same procedure as above and integrating out the original degrees of freedom, $c^{+}$and $c$, with a proper rescaling of the fields, we arrive at the dual-boson action

$$
\tilde{S}=-\sum_{\mathbf{k} \omega} \tilde{G}_{0}^{-1} \tilde{c}_{\mathbf{k} \omega \sigma}^{+} \tilde{c}_{\mathbf{k} \omega \sigma}-\frac{1}{2} \sum_{\mathbf{q} \nu} \tilde{W}_{0}^{-1} \tilde{\rho}_{\mathbf{q} \nu}^{*} \tilde{\rho}_{\mathbf{q} \nu}+\tilde{V},
$$

with the bare dual-fermion and dual-boson propagators, i.e. $\tilde{G}_{0}=G_{\mathrm{E}}-G_{\mathrm{imp}}$ and $\tilde{W}_{0}=W_{\mathrm{E}}-\mathcal{W}_{\mathrm{imp}}$, and with the dual interaction term $\tilde{V}$. We hereby also introduced the fermion $\left(G_{\mathrm{E}}\right)$ and boson $\left(W_{\mathrm{E}}\right)$ propagators for extended DMFT theory (E-DMFT) [50]. Note that, if we ignored interactions in the dual-boson action 
$(\tilde{V}=0)$, our approach would exactly reduce to the E-DMFT, where the fermionic Green's functions depends on the local solution only $\left(G_{\text {imp }}\right)$ and the bosonic lattice propagator merely depends on the impurity screened interaction $\left(\mathcal{W}_{\text {imp }}\right)$. In this case the E-DMFT self-consistent condition which connects the local part of the lattice propagators to the impurity ones gives solutions for the hybridization function $\Delta_{\omega}$ and the dynamical interactions $\mathcal{U}_{\nu}$.

Now the effective interaction in dual space not only consists of two-particle correlations ("square vertex") but also contains effective electron-boson correlations ("triangular vertex") which can be viewed as a result of partial bosonizations in the original diagrammatic series in powers of the interaction. In the standard dual-boson scheme one usually sums the ladder-type series of diagrams for the fermionic and bosonic propagators.

It is instructive to relate the dual-boson framework in its simplest approximation to the popular GW + DMFT scheme [51,52], see Figure 2. In this approach, one neglects the two-fermion vertex and in the same approximation sets the effective fermion-boson vertex to unity. The fermions always interact with bosons in this scheme, which makes the E-DMFT approximation (or zeroth-order dual-boson quasiparticles) not as good as the DMFT one. We use the lowest-order correction in the electron-boson interactions which corresponds to the second-order boson self-energy: $\tilde{\Pi}_{\mathbf{q} \nu}^{(2)}=-\sum_{\mathbf{k}, \omega} \tilde{G}_{\mathbf{k} \omega} \tilde{G}_{\mathbf{k}+\mathbf{q}, \omega+\nu}$. In addition to the local DMFT self-energy we will have the non-local contribution from effective electron-boson interactions. In the simplest RPA-like approximation this non-local part of GW+DMFT self-energy is equal to

$$
\tilde{\Sigma}_{\mathbf{k} \omega}^{G W}=\sum_{\mathbf{q} \nu} \tilde{G}_{\mathbf{k}-\mathbf{q}, \omega-\nu} \tilde{W}_{\mathbf{q} \nu}^{G W}, \quad \tilde{W}_{\mathbf{q} \nu}^{G W}=\tilde{U}_{\mathbf{q} \nu}\left[1-\left(\chi_{\nu}+\widetilde{\Pi}_{\mathbf{q} \nu}^{(2)}\right) \tilde{U}_{\mathbf{q} \nu}\right]^{-1}
$$

Let us point out two advantages of such a generalized GW + DMFT scheme: (a) the denominator of $\tilde{W}_{\mathbf{q} \nu}^{G W}$ contains the exact local susceptibility $\chi_{\omega}$, which improves the numerical accuracy of this approximation; (b) the lattice nonlocal interactions $U_{\mathbf{q}}$ needs to be corrected for the impurity local part $\mathcal{U}_{\nu}$ to give effective interactions $\tilde{U}_{\mathbf{q} \nu}$.

Despite the recent successes of the $G W+$ DMFT scheme, it does not provide a completely valid description of plasmons. This is due to an inconsistent treatment of the single- and two-particle properties, which breaks local charge conservation and gauge invariance. In case of a local and frequency-dependent self-energy in E-DMFT, vertex corrections from a local but frequency-dependent irreducible vertex are necessary to fulfill the Ward identity. In the dual-boson approach, this can be included via nonlocal polarization corrections, which are constructed diagrammatically. The resulting polarization vanishes in the long-wavelength limit at finite frequencies, as required by local-charge conservation [54]. It therefore becomes possible to study the effect of strong correlations on the plasmon spectra [53], showing that the two-particle excitations exhibit both renormalization of the dispersion and spectralweight transfer. This is similar to analogous interaction effects known from singleparticle excitations. Figure 3 shows the inverse dielectric function of two-dimensional surface plasmons in presence of long-range interaction $U_{\mathbf{q}}=U^{*}+V_{\mathbf{q}}$. For weak interaction one observes a broad particle-hole continuum and the expected $\sqrt{q}$ dependence of the plasmon dispersion at small $q$ in two dimensions. As the interaction increases, the plasmon dispersion $\omega_{p}^{2}(q) \approx \alpha V_{q}$ is renormalized. Two branches are clearly visible in the spectrum: The lower branch can be associated with particle-hole excitations between a Hubbard band and the quasiparticle peak, while the upper branch stems from excitations between Hubbard bands. Spectral weight is transferred from the lower to the upper branch as the interaction increases. Above a critical effective on-site interaction of $U^{*} \sim 2.4$, the system becomes a Mott insulator. In this state 


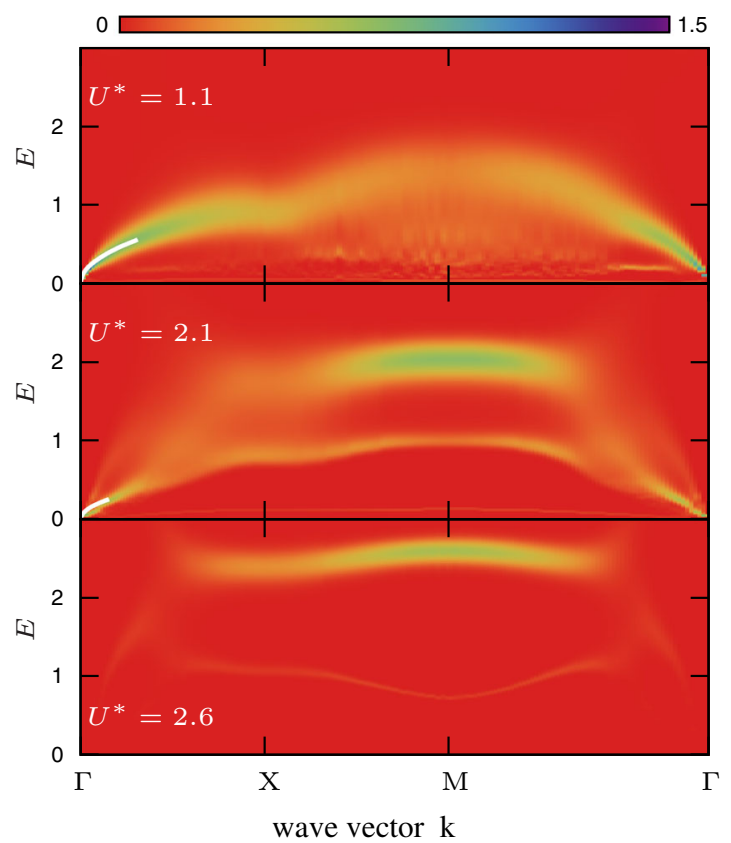

Fig. 3. Adapted from reference [53]: Inverse dielectric function as obtained by the dualboson approach for the extended two-dimensional Hubbard model with long-range Coulomb interaction as a function of momentum and energy. Results are shown for three different values of the effective on-site interaction $U^{*}$.

a two-particle excitation corresponds to the creation of a doublon and a holon. Such an excitation is highly localized, leading to a weakly dispersing band at energy $U^{*}$.

\section{Realistic DMFT for correlated materials}

The inclusion of nonlocal correlation effects using theoretical concepts as described, e.g., in the preceding section is an important route to go beyond the single-site DMFT. Another one is described in the following and may be termed "realistic DMFT". Its main idea is to combine the DMFT with density-functional theory (DFT), as will be discussed in Section 4.1 below, with the overall goal of quantitative predictions for specific materials rather than addressing model systems only. Already in seemingly standard-looking materials problems, multi-site and inhomogeneous aspects become relevant. Methodological aspects of the generalized real-space DFT+DMFT approach are mentioned in Section 4.2, and the metal-to-insulator transition in $\mathrm{V}_{2} \mathrm{O}_{3}$ as a concrete example is discussed in Section 4.3. A novel materials-design application of the given first-principles many-body theory is then discussed in Section 4.4 for the context of oxide heterostructures.

\subsection{Combining DFT with DMFT}

The promotion of DMFT to the realistic level by an adequate merging with electronic structure theory in DFT has been envisaged already early on [18]. While the study of model Hamiltonians is a very important research field in order to investigate and reveal key processes in correlated electron systems, concrete materials problems often 
harbor a vast number of relevant characteristics that are hard to condense into a tailored (minimal) model problem from the start. Low-symmetry crystal structure, multi-orbital manifolds, intricate crystal-field effects, structural distortions or sophisticated screening processes are only a few features that may render an approach within a first-principles many-body scheme favorable [50].

Since DMFT builds up on a tight-binding perspective with well-localized orbitals to represent interacting electron states, the linear-muffin-tin-orbital (LMTO) [2] scheme was the first natural Kohn-Sham-based DFT framework to be allied with the many-body method $[55,56]$. Thereby, the LMTO orbitals (or parts of it) associated with selected states, e.g., a sub-manifold of the $3 d$ shell of a transitionmetal ion, serve as the so-called correlated subspace. A different combinational route, building up on the Korringa-Kohn-Rostocker method for the Kohn-Sham problem, has a similar identification of the correlated states [57]. In order to utilize bandtheory approaches which employ a plane-wave basis, the notion of local orbitals has to be introduced. The spread of an inserted common LCAO basis is usually too large, but Wannier-function methodologies, e.g., in the maximally-localized form [58], are well suited. Thus using Wannier functions as a generic interface [59] allowed to open DFT+DMFT calculations to the widespread plane-wave community. The construction of explicit special-featured Wannier functions for certain bands can sometimes be numerically cumbersome, especially for the case of low crystal symmetries and/or large supercells. An efficient alternative is the projected-local-orbital formalism [60,61] which uses a simple projection of Kohn-Sham states onto localized orbitals. This appears quite flexible and frees the correlated subspace from a direct band association. The correlated subspace should be quite generally be understood as a quantum-numbered real-space region where correlated electrons are most likely hosted. That space surely connects to the DFT charge density, but not necessarily needs to originate from a one-to-one mapping to a certain band dispersion. Once a correlated subspace is selected, quantum impurity solvers based, e.g., on quantum Monte Carlo, Exact Diagonalization, etc. yield the DMFT impurity solution.

So far we only dealt with the interface from DFT to DMFT, i.e., the full calculation employs the downfolding of a converged Kohn-Sham cycle via the named interfacing to a kinetic Hamiltonian that is then treated together with a suitable interacting Hamiltonian. This so-called "one-shot" or "post-processing" approach has been the state of the art for several years. Its reliable to examine many spectral or local-moment properties [59,62-64]. However, since the realistic band-theory part and the interacting many-body part form the framework on equal footing, there has to be in addition an interfacing from DMFT to DFT to close a self-consistency loop. The charge self-consistent version of the method suits that goal. It computes an update of the, now correlated, charge density subject to the many-body self-energies. Hence there is an upfolding of the correlation effects to the complete Kohn-Sham (Bloch) Hilbert space. From the new charge density, a novel effective-single-particle potential for the next Kohn-Sham step is extracted and hence the full DFT+DMFT cycle [57,65-67] is closed (see Fig. 4). Using the normalized projections $\bar{P}$ between Bloch space and correlated subspace [60], the key equations governing the down- and upfold interfacing read

$$
\begin{aligned}
& G_{m m^{\prime}}^{\mathbf{R}, \mathrm{imp}}(\omega)=\sum_{\mathbf{k},\left(\nu \nu^{\prime}\right) \in \mathcal{W}} \bar{P}_{m \nu}^{\mathbf{R}}(\mathbf{k}) G_{\nu \nu^{\prime}}^{\mathrm{bloch}}(\mathbf{k}, \omega) \bar{P}_{\nu^{\prime} m^{\prime}}^{\mathbf{R} *}(\mathbf{k}), \\
& \Delta \Sigma_{\nu \nu^{\prime}}^{\mathrm{bloch}}(\mathbf{k}, \omega)=\sum_{\mathbf{R}, m m^{\prime}} \bar{P}_{\nu m}^{\mathbf{R} *}(\mathbf{k}) \Delta \Sigma_{m m^{\prime}}^{\mathrm{imp}}(\omega) \bar{P}_{m^{\prime} \nu^{\prime}}^{\mathbf{R}}(\mathbf{k}) .
\end{aligned}
$$

Here, at self-consistency, $G_{m m^{\prime}}^{\mathbf{R}, \text { imp }}$ is the impurity Green's function on site $\mathbf{R}$ with orbital indices $m, m^{\prime}$ and $\Delta \Sigma_{\nu \nu^{\prime}}^{\text {bloch }}$ is the $k$-dependent self-energy in Bloch 


\section{Density Functional Theory (DFT) + Dynamical Mean-Field Theory (DMFT)}

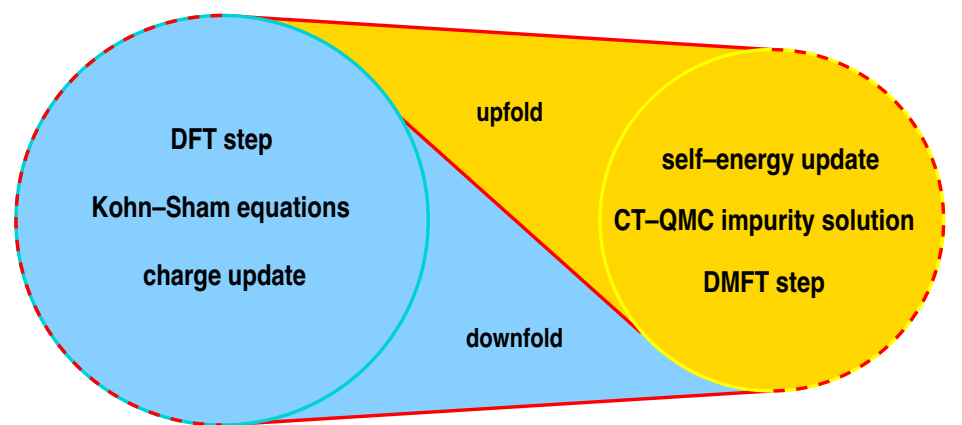

Fig. 4. Sketch of the charge self-consistent DFT+DMFT scheme pictured as a ratchet (for more details see e.g., [59]). It starts with a DFT step by solving the Kohn-Sham equations with respect to the effective lattice potential $v_{\text {eff }}$ and computing the resulting charge density. Downfolding to a correlated subspace defines a DMFT impurity problem and a solution step is undertaken, e.g., by using quantum Monte Carlo. The resulting self-energy is upfolded to the Bloch space and a novel $v_{\text {eff }}$, based on the correlation-modified charge density, constructed.

space after double-counting correction with band indices $\nu, \nu^{\prime}$. As for the correlated subspace, there is a choice for the range $\mathcal{W}$ of included Kohn-Sham bands in the calculation. The double-counting correction, similar as in static DFT $+\mathrm{U}[68$ 70], takes care of the fact that some correlations are already treated on the DFT level. Note that albeit the latter equations are written in spirit of single-site DMFT, the Bloch self-energy is indeed naturally $k$-dependent because of the $k$-dependence of the projections $\bar{P}$. In other words, since a given orbital has varying contribution to given Kohn-Sham bands in reciprocal space, so has the associated selfenergy. It is also instructive to reproduce the expression for the correlated charge density $\rho$, i.e.,

$$
\rho(\mathbf{r})=\sum_{\mathbf{k}, \nu \nu^{\prime}}\left\langle\mathbf{r} \mid \Psi_{\mathbf{k} \nu}\right\rangle\left(f\left(\tilde{\epsilon}_{\mathbf{k} \nu}\right) \delta_{\nu \nu^{\prime}}+\Delta N_{\nu \nu^{\prime}}(\mathbf{k})\right)\left\langle\Psi_{\mathbf{k} \nu^{\prime}} \mid \mathbf{r}\right\rangle
$$

where $\Psi$ denotes Kohn-Sham states, $f$ the associated Fermi function and $\Delta N$ is the DMFT self-energy correction term [59,60]. Without the latter, the charge density reduces to the original Kohn-Sham DFT form (and also realized in static DFT+U), resulting from contributions diagonal in the band index. However since a pure band picture is not vital in a many-body system and real-space excitations also matter, additional terms off-diagonal in the band index contribute in the correlated regime.

With this establishment of DFT+DMFT, correlated total energies become available and permit the investigation of, e.g., phase stabilities [67]. Note, finally, that this first-principles many-body scheme essentially works at finite temperature $T$. Electron states are subject to the full thermal impact, resulting, e.g., in a welldefined description of paramagnetism or the evaluation of coherence scales in electron transport.

This very brief sketch of the realistic-DMFT development described the matter on an informal level. Importantly, however, the approach may also be much more formally presented on the level of many-body functionals of Luttinger-Ward type. We refer to $[50,71]$ for detailed reviews on such formulations. 


\subsection{Real-space DFT + DMFT}

The correlated subspace in many quantum materials invokes not only a single lattice site. For instance, multi-component compounds often harbor various symmetry-equivalent or -inequivalent correlated transition-metal sites. But this is easy to handle, since an impurity problem can be defined for each symmetryinequivalent site, whereby the coupling of those arises via the DMFT self-consistency condition [47]. The self-energy of equivalent sites is determined for a representative site and transferred to the remaining sites via the proper symmetry relations.

This multi-site or real-space generalization of single-site DMFT, of course, also neglects in its realistic version explicit inter-site self-energy terms, i.e., explicit nonlocal correlations are absent. Yet especially fostered in the charge self-consistent framework, there is surely an implicit coupling, sufficient to sustain certain inter-site processes (cf. Sect. 2). Besides stoichiometric compounds with larger primitive cells, real-space DFT + DMFT is suited to investigate realistic defect physics in correlated materials, e.g., naturally occuring via doping or through intrinsic (point) defects. The research field of realistic defect-based many-body physics, albeit a pressing problem in many persistent quantum-materials challenges, is only about to receive more attention in recent years $[72,73]$. Let us note that of course also the standard cluster extensions to DMFT (see e.g., [25] for a review) can be allied with DFT in similar manners. But in the following subsections we will focus on multi-site applications.

The next two chapters deal with concrete applications using DFT+DMFT calculations based on the charge self-consistent combination of the mixed-basis pseudopotential method [74] for the DFT part and the CT-QMC method, as implemented in the TRIQS package $[75,76]$, for the DMFT impurity problem.

\subsection{The longstanding $\mathrm{V}_{2} \mathrm{O}_{3}$ problem as a concrete example}

For already decades, the vanadium sesquioxide $\mathrm{V}_{2} \mathrm{O}_{3}$ poses a famous challenge in the understanding of correlated materials [77]. The corundum compound has a canonical phase diagram $[78,79]$ that displays a paramagnetic-metallic, a paramagneticinsulating as well as an antiferromagnetic-insulating phase at finite $T$, depending on pressure or effective electron/hole doping with transition-metal substitutes, i.e., Cr or $\mathrm{Ti}$, on the vanadium sites. At ambient $T$ and pressure, the PM phase is the stable one for the stoichiometric compound. Along the crystallographic $c$-axis, pairs of $\mathrm{V}$ align, and the $a b$-plane is defined by a honeycomb lattice. A trigonal crystal field acts on the transition-metal site in $\mathrm{VO}_{6}$ octahedra. Thus the low-energy $t_{2 g}$ orbitals of the $\mathrm{V}(3 d)$ shell are split into an $a_{1 g}$ and two degenerate $e_{g}^{\prime}$ orbitals, providing a bandwidth $W \sim 2.6 \mathrm{eV}$ (see Fig. 5a). Formally, vanadium is in the oxidation state $3+$, i.e., a valence configuration $3 d^{2}$ holds. The transition-metal $t_{2 g}$ electrons are then also usually chosen to form the correlated subspace of $\mathrm{V}_{2} \mathrm{O}_{3}$. An interacting Hamiltonian of Slater-Kanamori form, parametrized by a Hubbard $U=5 \mathrm{eV}$ and a Hund's exchange $J_{\mathrm{H}}=0.93 \mathrm{eV}[62,80]$, is therein applied. Note that the corundum structure asks for two formula units in its primitive cell, i.e., there are four symmetry-equivalent $\mathrm{V}$ ions for multi-site DFT + DMFT.

From the $U / W$ ratio, the $\mathrm{V}_{2} \mathrm{O}_{3}$ system is in a strongly correlated regime, but the detailed mechanism of the metal-to-insulator transition (MIT) remains a matter of debate. Of course, as basic DMFT teaches us, at a large local interaction strength a condensed-matter electronic system will become Mott insulating. But this statement is often not sufficient to explain or predict details of MITs in materials. The interplay of lattice structure, multi-orbital characteristics, defect properties and temperature in a realistic system, allows nature to realize quite intricate driving mechanisms. 

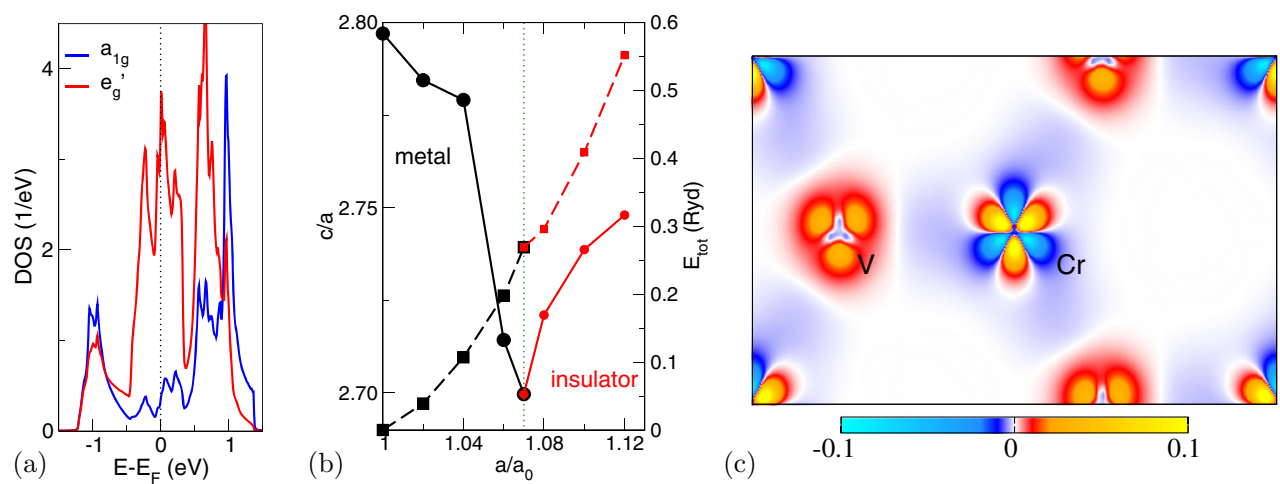

Fig. 5. Adapted from references [67,73]: Data for $\mathrm{V}_{2} \mathrm{O}_{3}$. (a) LDA low-energy density of states. (b) Left panel, full lines: relaxed $c / a$ ratio vs. lattice constant. Right panel, dashed lines: total energy vs. lattice constant. Black/Red lines mark metal/insulator. (c) Difference of DFT+DMFT and LDA charge density in the $a b$-plane. Note the charging of $\mathrm{V}$ by $\mathrm{Cr}$ due to electronic correlations.

Let us in the following restrict the discussion to the paramagnetic regime. Experimentally, in essence, Cr-doping drives $\mathrm{V}_{2} \mathrm{O}_{3}$ insulating at constant temperature, accompanied by a subtle lattice expansion [81] as well as orbital polarization towards $e_{g^{\prime}}$ [82]. First-principles DFT [83-87] and DFT + DMFT [62,67,73,88-92] studies approached the problem. Originally, the focus of most of the theoretical works is on the "proper averaged" electronic properties, i.e., leaving the very details of the (electron-)lattice degrees of freedom and the doping/defect physics aside. Then, whereas conventional DFT surely fails to describe a MIT, "one-shot" multi-orbital DFT + DMFT indeed suggests a transition. But it does so on the basis of experimental lattice data for the metallic and insulating phase $[62,88,89]$, while keeping the $\mathrm{V}_{2} \mathrm{O}_{3}$ stoichiometry in the calculation. The orbital polarization is explained by a crystal-field enhancement due to electronic correlations [88,89].

Recently original [67] and follow-up charge self-consistent DFT + DMFT [73,91, 92] suggests some modifications to this picturing. The correlation-enhanced orbital polarization in stoichiometric $\mathrm{V}_{2} \mathrm{O}_{3}$ (with or without expanded lattice) is predicted to be much weaker. This theoretical forecast is indeed verified in very recent angleresolved photoemission experiments [93]. Additionally, structural relaxation of the $c / a$ ratio in the correlated system [67] exhibits the subtle electron-lattice coupling, marking strong $c / a$ changes close to the MIT (see Fig. 5b). Related formal divergences of compressibilities near a Mott transition where before only studied within a model context [94]. A more thorough treating of $\mathrm{Cr}$ doping within an explicit supercell approach rendered it furthermore obvious that the effect of the chromium dopants may not only be reduced to a sole lattice-expansion effect. The valence of $\mathrm{Cr}$ has an additional electron and apparently electron-dopes the neighboring $\mathrm{V}$ sites in an orbital-selective manner (cf. Fig. 5c), giving rise to the measured orbital polarization [73]. Note that is has indeed been shown [95], that applied pressure on insulating Cr-doped $\mathrm{V}_{2} \mathrm{O}_{3}$ renders the system metallic without substantial changes in the orbital polarization.

A complete understanding of the MIT in vanadium sesquioxide is still missing, but DFT + DMFT was so far very successful in sheding light on various important features. There is evidence that a full solution asks for the inclusion of correlations, coupling to the lattice and defect physics on a close-to equal footing. 


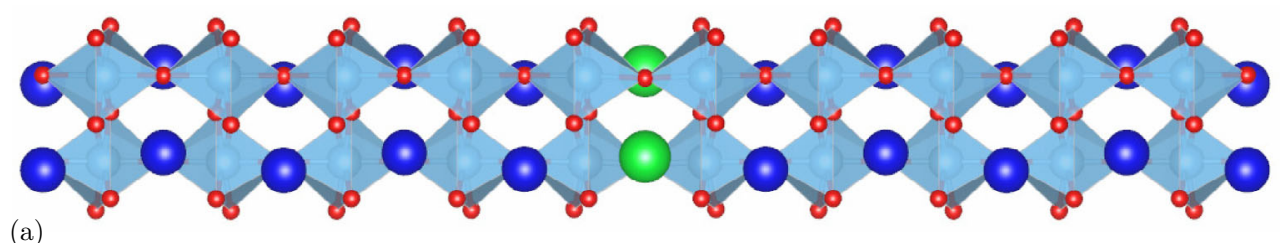

(a)
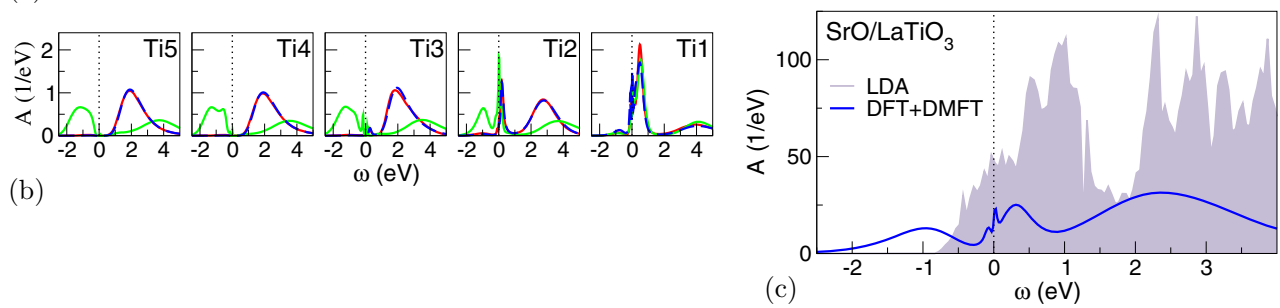

Fig. 6. Adapted from reference [101]: DFT + DMFT description of $\delta$-doped $\mathrm{LaTiO}_{3}$ $(T=145 \mathrm{~K})$. (a) Structurally relaxed supercell with La (blue), Ti (grey), O (red) and $\mathrm{Sr}$ (green) atoms, $c$-axis is aligned horizontally. (b) Local Ti1-5 spectral functions for the effective $t_{2 g}$ orbitals. Ti locations correspond with (a), i.e., Ti1/5 is closest/farest to the interface. (c) Total spectral function compared to the LDA result.

\subsection{Oxide heterostructures: $\delta$-doping of titanate Mott insulators}

The research field of oxide heterostructures has become a prominent one in condensed matter physics (see e.g., [96-98] for reviews). Materials design starting from metallic, band- or Mott-insulating transition-metal oxides can lead to emergent physics due to interfacing, e.g., to the formation of two-dimensional electron systems. As electronic correlations come easy with such oxides, there is the chance of a new era of realistic many-body physics with possibly novel exotic phases and various engineering options.

As a highlighting and instructive example, we will show how DFT+DMFT can help to understand or even predict the complex electronic structure in oxide Mott insulators doped by heterostructuring. Experimental work on oxide heterostructures grown with molecular-beam epitaxy revealed puzzling electron states in rare-earth $(R)$ titanates $R \mathrm{TiO}_{3}$ upon $\delta$-doping $[99,100]$, i.e., monolayer doping, with SrO. The bulk distorted perovskites are formal $\mathrm{Ti}\left(3 d^{1}\right)$ systems, whereby the single electron occupies the effective $t_{2 g}$ subshell. The well-defined $\delta$-doping of such Mott insulators is free from disorder effects or screening-length ambiguities. Unusual metallicity as well as complex magnetic response has been detected.

By employing a large supercell (see Fig. 6a) to incorporate the regions close and far away from the doping layer, real-space DFT + DMFT calculations including charge self-consistency indeed reveal a demanding electronic structure [101]. We focus in the following on the doped $\mathrm{LaTiO}_{3}$ system described by a 100 -atom primitive cell, with lattice parameters from experiment [102] and DFT-relaxed atomic positions. There are $20 \mathrm{Ti}$ ions, forming the 5 symmetry-inequivalent classes Ti1-5 resembling the different $\mathrm{TiO}_{2}$ layers counted from the SrO doping layer. The correlated subspace consists of the effective $t_{2 g}$ manifold formed by all the Ti sites and obtained by the projected-local-orbital formalism. Again a Slater-Kanamori local-Hamiltonian form is put into practice, with $U=5 \mathrm{eV}$ and $J_{\mathrm{H}}=0.64 \mathrm{eV}$, as proper for titanates [64].

Of course in DFT, $\delta$-doped $\mathrm{LaTiO}_{3}$ is metallic (as the stoichiometric compound). As displayed in Figure 6c, a metallic state is also detected within DFT + DMFT for the overall system, but showing a renormalized quasiparticle peak as well as spectralweight transfer to a lower Hubbard band. Moreover the calculations uncover a rich 
correlated electronic structure with respect to the distance from the $\mathrm{SrO}$ doping layer (see Fig 6b). Far from there, indeed a Mott-insulating state is stabilized, with nearly complete orbital polarization to one effective $t_{2 g}$ level. The $\mathrm{TiO}_{2}$ layer next to SrO harbors a nearly-orbital-polarized metallic state that appears Fermi-liquid like [101]. The electrons in the second $\mathrm{TiO}_{2}$ layer between those two states form an orbital-polarized doped-Mott state with metallic response but seemingly unusuallarge electron-electron scattering. In a recent advancement of these investigations to the case of $\delta$-doped $\mathrm{SmTiO}_{3}$, a rare-earth titanate close to an antiferromagneticferromagnetic instability, non-Fermi-liquid behavior and fingerprints of a pseudogap have been revealed [103].

The findings render it obvious that not only a well-defined doped-Mott system with layer-dependent electron characteristics may be realized by oxide heterostructuring. A further (theoretical) engineering of those characteristics appears possible via, e.g., additional chemical dopants, strain, different layerings, etc., opening the possibility for the intriguing stabilization of known and/or exotic orders.

\section{Realistic nonlocal correlations: spin-polaron physics in $\mathrm{Na}_{x} \mathrm{CoO}_{2}$}

So far we discussed nonlocal self-energies $\Sigma(\mathbf{k}, \omega)$ in a model context and local selfenergies $\Sigma(\omega)$ for realistic cases. Due to complexity, realistic nonlocal correlations are (even) much more demanding. Nonetheless, we are able to report a realistic dualfermion study on the interaction of electrons and paramagnons in strongly correlated sodium cobaltate $\mathrm{Na}_{x} \mathrm{CoO}_{2}$, close the in-plane ferromagnetic order at $x=0.7$ [105]. Let us briefly summarize the problem and the obtained results. Sodium cobaltate consists of triangular $\mathrm{CoO}_{2}$ planes subject to strong electronic correlations, that are held together by $\mathrm{Na}$ ions in between (cf. Fig. 7a). As shown in Figure 7b, the resulting phase diagram upon variation of the sodium content $x$ is rather rich, and we are here focussing on the onset of in-plane ferromagnetism close to the band-insulating $x=1$ limit. While for smaller $x$ a single hole resides in the low-energy $\mathrm{Co}\left(t_{2 g}\right)$ shell, giving rise to Mott physics, at larger $x$ ferromagnetic fluctuations have their share on the electronic correlations. In a first step, we computed $q$-dependent dynamic lattice susceptibilities beyond the bubble approximations, i.e., included relevant vertex corrections [104]. The latter are based on the two-particle Green's function and the extraction of the local vertex function $[104,107]$. Figure $7 \mathrm{c}$ displays the obtained paramagnon dispersion close to the $\Gamma$ point in reciprocal space at higher doping $x$. This enhanced spin susceptibility close to $\Gamma$ should affect the one-particle spectral function, namely by a coupling between electrons and paramagnons. But as described in Section 3, such processes may just be described within the dual-fermion scheme. In short, the $k$-dependent dual self-energy can be expressed as

$$
\tilde{\Sigma}(\mathbf{k}, \omega)=\sum_{\alpha \nu \omega^{\prime} \mathbf{q}} a_{\alpha} \gamma_{\omega \omega^{\prime}}^{\nu \alpha} \tilde{G}(\mathbf{k}+\mathbf{q}, \omega+\nu) \tilde{\chi}_{\nu}^{\omega^{\prime}}(\mathbf{q}) \hat{\Gamma}_{\omega^{\prime} \omega}^{\nu \alpha}(\mathbf{q}),
$$

whereby $\alpha$ differs spin and charge channel, $\omega / \nu$ are fermionic/bosonic Matsubara frequencies, $\chi$ is the dual particle-hole bubble, $\tilde{G}$ the dual Green's function, $\hat{\Gamma}$ the lattice vertex function and $\gamma$ the local irreducible vertex. Thus it indeed describes the self-energy building up from one-particle and two-particle objects.

Using a tailored one-band description of the low-energy cobaltate physics, we thus derived a realistic DFT-based dispersion, applied a proper Hubbard $U=5 \mathrm{eV}$ and solved for the local and nonlocal correlations by the elaborate dual-fermion framework (see [105] for details). And indeed, for $x=0.7$ an additional anti-bound state is detected close to $\Gamma$, split off from the renormalized quasiparticle dispersion 

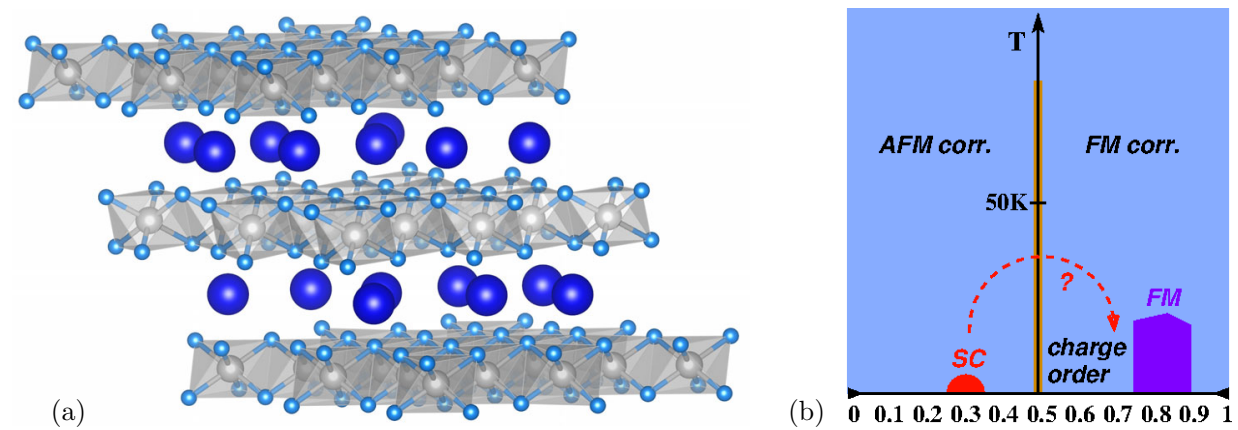

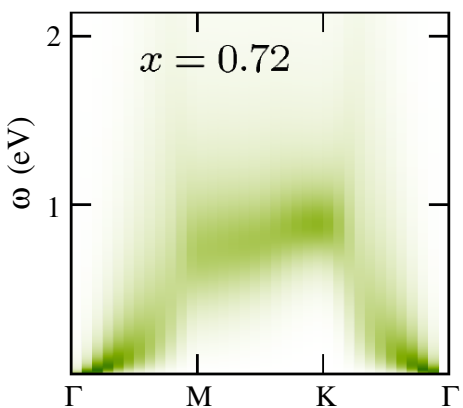

(c)

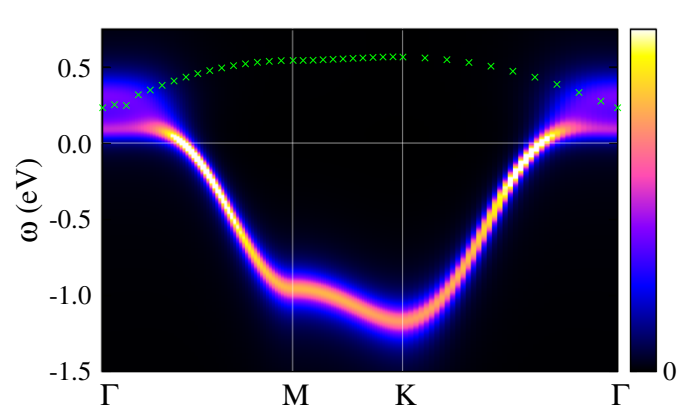

(d)

Fig. 7. Adapted from references $[104,105]$ : The $\mathrm{Na}_{x} \mathrm{CoO}_{2}$ system harboring electronparamagnon interactions. (a) Crystal structure with $\mathrm{Na}$ (large blue), Co (grey) and O (small blue) ions. (b) Sketched $x-T$ in-plane phase diagram, including the open issue [106] on the location of the superconducting region upon water intercalation. (c) DMFT-based dynamic in-plane spin susceptibility obtained by a CT-QMC impurity solver for $x=0.72$ including vertex corrections. (d) Effective one-band dispersion for $x=0.7$ resulting from the dualfermion method in the ladder approximation. Green crosses mark a model spin-polaron dispersion (see Ref. [105] for details).

(cf. Fig. 7c). Again in terms of physics, it corresponds to the interaction of renormalized electrons with strong ferromagnetic fluctuations. The resulting emerging excitation is a so-called spin polaron and experimental fingerprints thereof have indeed been detected in optics measurements by Wan et al. [108]. Hence besides refinements of the DMFT-determined electronic spectrum, the inclusion of nonlocal correlations may be important to reveal more complex excitations with possible relevance for future materials science.

\section{Perspectives}

The extremely diverse and fascinating physical properties of classical and modern condensed-matter systems, ranging from simple metals (as, e.g., $\mathrm{Al}$ ) over semiconductors (such as GaAs), itinerant ferromagnets ( $\mathrm{Fe}$ ), Mott or charge-transfer insulators $(\mathrm{NiO})$, to high- $T_{\mathrm{C}}$ superconductors $\left(\mathrm{La}_{1-x} \mathrm{Ba}_{x} \mathrm{CuO}_{4}\right)$ and graphene, to name a few examples only, are to a large extent determined by their electronic structure. It is clear that a realistic modeling of the interacting electron gas in a solid that covers materials of such different kind in a coherent theoretical framework represents a highly ambitious project. In fact, this project goes on for decades and is by no means finished. It is triggered by an ever-improving set of sophisticated experimental 
techniques, in particular by the various breakthroughs in high-resolution photoemission spectroscopy, which is the technique that gives the most direct access to the electronic structure. Also the discovery of new materials or the completely revised understanding of "well-known" ones pushes this project. Predictive power is the regulative idea, which drives the research in this field, since the ultimate goal of any theory should be to make measurable predictions, as precise as possible, without any a posteriori input, and starting only from general information, such as chemical composition.

With the focus on strongly correlated electron systems, the next level in this endeavor is reached. Typically, the electronic properties of these correlated materials are extremely susceptible to all kinds of perturbations. This may lead to a substantially increased complexity of the essential physics. A related characteristics of strongly correlated systems is that different electronic mechanisms are at work, favoring phases with different types of local, short- or long-range correlations. These compete or cooperate with each other at low temperatures and on tiny energy scales. Besides the bare energy scales, given by the one-particle or interaction parameters, also new and typically much smaller energy scales are generated by correlations. This can give rise to involved thermodynamical phase diagrams, already for model systems as we have seen in Section 2 where single-site screening effects, nonlocal magnetic correlations and geometrical frustration compete with each other.

Understanding strongly correlated systems thus requires (i) nonperturbative approaches to tackle the correlation problem and (ii) highly accurate tools to describe the chemical and geometrical aspects and to compute one-particle and interaction matrix elements. Using dynamical mean-field theory, in a proper combination with refined concepts of band-structure theory, substantial progress could be made in the recent past.

While the separate potentials of DFT and of DMFT themselves are not at all fully exhausted, the versatile usability and the robustness of the band-structure and of the mean-field concept make the combination of DFT with DMFT an almost ideal approach to cover complex materials with strong correlations. Examples for involved multi-orbital physics have been discussed in Sections 4.3 and 5. In this context, there are several possibly important issues that must be accounted for and that can be covered in fact, such as low-symmetry crystal structures, intricate crystal-field effects, structural distortions, sophisticated screening processes, etc. This also includes lack of translational symmetry, see Section 4.4. Inhomogeneous correlated systems, such as surfaces and interfaces, can be studied with the real-space variant of DFT+DMFT (Sect. 4.2). This is important to make close quantitative contact with highly surfacesensitive photoemission spectroscopy.

To advance DMFT to a first-principles approach with predictive power for real materials, spanning traditionally studied and modern strongly correlated materials, special attention must be spent on the interface with density-functional theory. The interface is crucial as the nonlocal concepts from band theory must be combined with the locality paradigm of DMFT. Related to this is the choice of a proper oneparticle basis set, the definition of a physically motivated "correlated subspace" and the forth and back switching ("up and down folding") between the DMFT treatment of a lattice model with local interactions in the correlated subspace on the one hand, and the effective one-particle multiple-scattering problem in the entire orbital space on the other. Actually, this full complexity shows up when running through the "big self-consistency loop". This comes in addition to the internal loops that are characteristic for DMFT, which self-consistently maps onto an effective impurity problem, and for DFT, which self-consistently maps onto the homogeneous electron gas. The big loop or charge-self-consistency provides the necessary feedback of the 
correlation-modified charge density on the effective one-particle potential (cf. Sect. 4.1). Also in this respect, we have witnessed great progress in the recent years.

What is the to-do list for future developments? This question has at least two lines of possible answers. First, the present activities must be continued to further evolve the DFT+DMFT approach. This includes the development of better impurity solvers, which are able to access lower temperatures and to treat impurity models with more and more orbitals, and which give reliable real-frequency information. Improved quantum Monte-Carlo techniques (along the lines of Ref. [22]), approaches based on the time-dependent density-matrix renormalization group [109] or independent novel ideas [24] are promising. Second, extensions of the theory which incorporate the feedback of nonlocal correlations on the self-energy must be advanced (see our discussion in Sect. 3). In addition, further extensions are necessary to treat systems with nonlocal interactions. There are different diagrammatic or Green's-function-based approaches which are worth pursuing (cf. Sect. 3), but it is probably fair to say that the present theoretical ideas are far from providing an answer that is conceptually as clear as DMFT has given to the question of how to treat local correlations and local interactions. A third problem is the interface to the experiment. Besides static thermodynamical properties, the central quantity of DFT+DMFT is the one-particle spectral function which, however, is not directly observable. A quantitative prediction of photoemission spectra rather requires a proper inclusion of transition-matrix elements, an important issue that is in most cases set aside, unnecessarily. A challenge, on the other hand, is the development of a realistic theory of time-dependent (pump-probe) photoemission from correlated materials (see Ref. [110] for first attempts). While nonequilibrium DMFT [111] is already on the market, a nonequilibrium DFT+DMFT, however, is not yet in sight.

Finally, there are some future challenges which are of a more fundamental character: One example is the so-called double-counting problem, i.e., an incorrect double counting of interactions, once on some average level within the local-density approximation of DFT or variants of the LDA and once more, and more explicit, in the correlated lattice model to which DMFT is applied. While double-counting "corrections" are regularly used in practical DFT+DMFT calculations and while in most cases this is fully sufficient from a pragmatic point of view, all of them lack a thorough theoretical foundation. The GW+DMFT approach elegantly circumvents the double-counting problem, but eliminates the DFT-part of DFT+DMFT altogether, and it is surely questionable whether $\mathrm{GW}$ as a starting point is as reliable and versatile as the more traditional DFT.

Another fundamental critique targets the DMFT as such, and also its cluster or diagrammatic extensions, as these are mean-field approaches in the end and thus also share some typical problems associated with the mean-field concept. Critical phenomena, for example, are not easily described correctly when starting from the local DMFT. Also the possibility for nonunique solutions, resulting from the nonlinearity of the DMFT self-consistency (or mean-field) equation, must be seen as problematic from a fundamental quantum-statistical point of view - although this is often seen as advantageous and exploited in practice. Finally, it is by no means obvious that the central quantity of DMFT, the one-particle Green's function, deserves a distinguished role. Competing theories start from different observables, such as higher-order Green's functions, or even the many-body wave function, constrained, e.g., to some "physical corner" of the Hilbert space [112], or the reduced one-particle density matrix. First attempts to clarify the mutual relation between the different concepts appear very promising [113]. Up to now, however, there is no working alternative to the DFT+DMFT concept when addressing the fascinating world of correlation effects in real materials. 
We would like to thank M.W. Aulbach, L. Boehnke, D. Grieger, H. Hafermann and E.G.C.P. van Loon for the intense and fruitful cooperation over the years. Financial support of this work by the Deutsche Forschungsgemeinschaft through the Forschergruppe FOR 1346 (project P1) is gratefully acknowledged.

Open Access This is an Open Access article distributed under the terms of the Creative Commons Attribution License (http://creativecommons.org/licenses/by/4.0), which permits unrestricted use, distribution, and reproduction in any medium, provided the original work is properly cited.

\section{References}

1. P.M. Marcus, J.F. Janak, A.R. Williams, eds., Computational Methods in Band Theory (Plenum, New York, 1971)

2. O.K. Andersen, Phys. Rev. B 12, 3060 (1975)

3. P. Blaha, K. Schwarz, P. Sorantin, S.B. Trickey, Comput. Phys. Commun. 59, 399 (1990)

4. W.H. Butler, P.H. Dederichs, A. Gonis, R.L. Weaver, Applications of Multiple Scattering Theory to Material Science (Materials Reserach Society, Pittsburg Penn., 1992)

5. P. Hohenberg, W. Kohn, Phys. Rev. 136, 864 (1964)

6. W. Kohn, L.J. Sham, Phys. Rev. 140, 1133 (1965)

7. R.O. Jones, O. Gunnarsson, Rev. Mod. Phys. 61, 689 (1989)

8. M. Donath, P.A. Dowben, W. Nolting, eds., Magnetism and Electronic Correlations in Local-Moment Systems: Rare-earth Elements and Compounds (World Scientific, Singapore, 1998)

9. N.F. Mott, Metal-Insulator Transitions, 2nd edn. (Taylor and Francis, London, 1990)

10. J. Orenstein, A.J. Millis, Science 288, 468 (2000)

11. A.A. Abrikosow, L.P. Gorkov, I.E. Dzyaloshinski, Methods of Quantum Field Theory in Statistical Physics (Prentice-Hall, New Jersey, 1964)

12. S. Hüfner, ed., Very High Resolution Photoelectron Spectroscopy (Springer, New York, 2007)

13. M.C. Gutzwiller, Phys. Rev. Lett. 10, 159 (1963)

14. J. Hubbard, Proc. R. Soc. London A 276, 238 (1963)

15. J. Kanamori, Prog. Theor. Phys. (Kyoto) 30, 275 (1963)

16. W. Metzner, D. Vollhardt, Phys. Rev. Lett. 62, 324 (1989)

17. A. Georges, G. Kotliar, Phys. Rev. B 45, 6479 (1992)

18. A. Georges, G. Kotliar, W. Krauth, M.J. Rozenberg, Rev. Mod. Phys. 68, 13 (1996)

19. G. Kotliar, D. Vollhardt, Phys. Today 57, 53 (2004)

20. M. Caffarel, W. Krauth, Phys. Rev. Lett. 72, 1545 (1994)

21. R. Bulla, T.A. Costi, T. Pruschke, Rev. Mod. Phys. 80, 395 (2008)

22. E. Gull, A. Millis, A. Lichtenstein, A. Rubtsov, M. Troyer, P. Werner, Rev. Mod. Phys. 83, 349 (2011)

23. M. Ganahl, P. Thunström, F. Verstraete, K. Held, H.G. Evertz, Phys. Rev. B 90, $045144(2014)$

24. Y. Lu, M. Höppner, O. Gunnarsson, M.W. Haverkort, Phys. Rev. B 90, 085102 (2014)

25. T. Maier, M. Jarrell, T. Pruschke, M.H. Hettler, Rev. Mod. Phys. 77, 1027 (2005)

26. A. Toschi, A.A. Katanin, K. Held, Phys. Rev. B 75, 045118 (2007)

27. A.N. Rubtsov, M.I. Katsnelson, A.I. Lichtenstein, Phys. Rev. B 77, 033101 (2008)

28. A.N. Rubtsov, M.I. Katsnelson, A.I. Lichtenstein, Ann. Phys. 327, 1320 (2012)

29. P.W. Anderson, Phys. Rev. 124, 41 (1961) 
30. Z. Fisk, H. Ott, T.M. Rice, J. Smith, Nature 320, 124 (1986)

31. H. Tsunetsugu, M. Sigrist, K. Ueda, Rev. Mod. Phys. 69, 809 (1997)

32. S. Capponi, F.F. Assaad, Phys. Rev. B 63, 155114 (2001)

33. J.R. Schrieffer, P.A. Wolff, Phys. Rev. 149, 491 (1966)

34. S. Doniach, Physica 91B, 321 (1977)

35. H.v. Löhneysen, A. Rosch, M. Vojta, P. Wölfle, Rev. Mod. Phys. 79, 1015 (2007)

36. M.A. Ruderman, C. Kittel, Phys. Rev. 96, 99 (1954)

37. J. Kondo, Prog. Theor. Phys. 32, 37 (1964)

38. A.C. Hewson, The Kondo Problem to Heavy Fermions, Cambridge Studies in Magnetism (Cambridge Universiy Press, Cambridge, 1997)

39. M.W. Aulbach, F.F. Assaad, M. Potthoff, Phys. Rev. B 92, 235131 (2015)

40. A.N. Rubtsov, V.V. Savkin, A.I. Lichtenstein, Phys. Rev. B 72, 035122 (2005)

41. P. Werner, A. Comanac, L.de' Medici, M. Troyer, A.J. Millis, Phys. Rev. Lett. 97, $076405(2006)$

42. Y. Motome, K. Nakamikawa, Y. Yamaji, M. Udagawa, Phys. Rev. Lett. 105, 036403 (2010)

43. R. Ballou, C. Lacroix, M.D. Nunez Regueiro, Phys. Rev. Lett. 66, 1910 (1991)

44. A. Benlagra, L. Fritz, M. Vojta, Phys. Rev. B 84, 075126 (2011)

45. S. Hayami, M. Udagawa, Y. Motome, J. Phys. Soc. Jpn. 80, 073704 (2011)

46. S. Hayami, M. Udagawa, Y. Motome, J. Phys. Soc. Jpn. 81, 103707 (2012)

47. M. Potthoff, W. Nolting, Phys. Rev. B 55, 2741 (1997)

48. I. Titvinidze, A. Schwabe, N. Rother, M. Potthoff, Phys. Rev. B 86, 075141 (2012)

49. M.W. Aulbach, I. Titvinidze, M. Potthoff, Phys. Rev. B 91, 174420 (2015)

50. G. Kotliar, S.Y. Savrasov, K. Haule, V.S. Oudovenko, O. Parcollet, C.A. Marianetti, Rev. Mod. Phys. 78, 865 (2006)

51. S. Biermann, F. Aryasetiawan, A. Georges, Phys. Rev. Lett. 90, 086402 (2003)

52. P. Sun, G. Kotliar, Phys. Rev. B 66, 085120 (2002)

53. E.G.C.P. van Loon, H. Hafermann, A.I. Lichtenstein, A.N. Rubtsov, M.I. Katsnelson, Phys. Rev. Lett. 113, 246407 (2014)

54. E.A. Stepanov, E.G.C.P. van Loon, A.A. Katanin, A.I. Lichtenstein, M.I. Katsnelson, A.N. Rubtsov, Phys. Rev. B 93, 045107 (2016)

55. V.I. Anisimov, A.I. Poteryaev, M.A. Korotin, A.O. Anokhin, G. Kotliar, J. Phys.: Condens. Matter 9, 7359 (1997)

56. A.I. Lichtenstein, M. Katsnelson, Phys. Rev. B 57, 6884 (1998)

57. J. Minár, L. Chioncel, A. Perlov, H. Ebert, M.I. Katsnelson, A.I. Lichtenstein, Phys. Rev. B 72, 045125 (2005)

58. N. Marzari, A.A. Mostofi, J.R. Yates, I. Souza, D. Vanderbilt, Rev. Mod. Phys. 84, 1419 (2012)

59. F. Lechermann, A. Georges, A. Poteryaev, S. Biermann, M. Posternak, A. Yamasaki, O.K. Andersen, Phys. Rev. B 74, 125120 (2006)

60. B. Amadon, F. Lechermann, A. Georges, F. Jollet, T.O. Wehling, A.I. Lichtenstein, Phys. Rev. B 77, 205112 (2008)

61. V.I. Anisimov, D.E. Kondakov, A.V. Kozhevnikov, I.A. Nekrasov, Z.V. Pchelkina, J.W. Allen, S.K. Mo, H.D. Kim, P. Metcalf, S. Suga, et al., Phys. Rev. B 71, 125119 (2005)

62. K. Held, G. Keller, V. Eyert, D. Vollhardt, V.I. Anisimov, Phys. Rev. Lett. 86, 5345 (2001)

63. A.I. Lichtenstein, M. Katsnelson, G. Kotliar, Phys. Rev. Lett. 87, 067205 (2001)

64. E. Pavarini, S. Biermann, A. Poteryaev, A.I. Lichtenstein, A. Georges, O.K. Andersen, Phys. Rev. Lett. 92, 176403 (2004)

65. S.Y. Savrasov, G. Kotliar, E. Abrahams, Nature 410, 793 (2001)

66. L.V. Pourovskii, B. Amadon, S. Biermann, A. Georges, Phys. Rev. B 76, 235101 (2007)

67. D. Grieger, C. Piefke, O.E. Peil, F. Lechermann, Phys. Rev. B 86, 155121 (2012)

68. V.I. Anisimov, J. Zaanen, O.K. Andersen, Phys. Rev. B 44, 943 (1991) 
69. V.I. Anisimov, I.V. Solovyev, M.A. Korotin, M.T. Czyżyk, G.A. Sawatzky, Phys. Rev. B 48, 16929 (1993)

70. M.T. Czyżyk, G.A. Sawatzky, Phys. Rev. B 49, 14211 (1994)

71. A. Georges, Strongly Correlated Electron Materials: Dynamical mean-field Theory and Electronic Structure, Lectures on the Physics of Highly Correlated Electron Systems VIII, AIP Conf. Proc. 715, 3 (2004)

72. F. Lechermann, L. Boehnke, D. Grieger, C. Piefke, Phys. Rev. B 90, 085125 (2014)

73. D. Grieger, F. Lechermann, Phys. Rev. B 90, 115115 (2014)

74. S.G. Louie, K.M. Ho, M.L. Cohen, Phys. Rev. B 19, 1774 (1979)

75. O. Parcollet, M. Ferrero, T. Ayral, H. Hafermann, I. Krivenko, L. Messio, P. Seth, Comput. Phys. Commun. 196, 398 (2015)

76. P. Seth, I. Krivenko, M. Ferrero, O. Parcollet, Comput. Phys. Commun. 200, 274 (2016)

77. C. Castellani, C.R. Natoli, J. Ranninger, Phys. Rev. B 18, 4945 (1978)

78. D.B. McWhan, T.M. Rice, J.B. Remeika, Phys. Rev. Lett. 23, 1384 (1969)

79. D.B. McWhan, J.B. Remeika, T.M. Rice, W.F. Brinkman, J.P. Maita, A. Menth, Phys. Rev. Lett. 27, 941 (1971)

80. A.E. Antipov, I.S. Krivenko, V.I. Anisimov, A.I. Lichtenstein, A.N. Rubtsov, Phys. Rev. B 86, 155107 (2012)

81. P.D. Dernier, J. Phys. Chem. Solids 31, 2569 (1970)

82. J.H. Park, L.H. Tjeng, A. Tanaka, J.W. Allen, C.T. Chen, P. Metcalf, J.M. Honig, F.M.F. de Groot, G.A. Sawatzky, Phys. Rev. B 61, 11506 (2000)

83. L.F. Mattheiss, J. Phys.: Condens. Matter 6, 6477 (1994)

84. S.Y. Ezhov, V.I. Anisimov, D.I. Khomskii, G.A. Sawatzky, Phys. Rev. Lett. 83, 4136 (1999)

85. I.S. Elfimov, T. Saha-Dasgupta, M.A. Korotin, Phys. Rev. B 68, 113105 (2003)

86. V. Eyert, U. Schwingenschlögl, U. Eckern, Europhys. Lett. 70, 782 (2005)

87. Y. Guo, S.J. Clark, J. Robertson, J. Chem. Phys. 140 (2014)

88. G. Keller, K. Held, V. Eyert, D. Vollhardt, V.I. Anisimov, Phys. Rev. B 70, 205116 (2004)

89. A.I. Poteryaev, J.M. Tomczak, S. Biermann, A. Georges, A.I. Lichtenstein, A.N. Rubtsov, T. Saha-Dasgupta, O.K. Andersen, Phys. Rev. B 76, 085127 (2007)

90. M. Sandri, M. Capone, M. Fabrizio, Phys. Rev. B 87, 205108 (2013)

91. X. Deng, A. Sternbach, K. Haule, D. Basov, G. Kotliar, Phys. Rev. Lett. 113, 246404 (2014)

92. I. Leonov, V.I. Anisimov, D. Vollhardt, Phys. Rev. B 91, 195115 (2015)

93. I. Lo Vecchio, J.D. Denlinger, O. Krupin, B.J. Kim, P.A. Metcalf, S. Lupi, J.W. Allen, A. Lanzara, Phys. Rev. Lett. 117, 166401 (2016)

94. S.R. Hassan, A. Georges, H.R. Krishnamurthy, Phys. Rev. Lett. 94, 036402 (2005)

95. F. Rodolakis, J.P. Rueff, M. Sikora, I. Alliot, J.P. Itié, F. Baudelet, S. Ravy, P. Wzietek, P. Hansmann, A. Toschi, et al., Phys. Rev. B 84, 245113 (2011)

96. P. Zubko, S. Gariglio, M. Gabay, P. Ghosez, J.M. Triscone, Annu. Rev. Condens. Matter Phys. 2, 141 (2011)

97. H.Y. Hwang, Y. Iwasa, M. Kawasaki, B. Keimer, N. Nagaosa, Y. Tokura, Nat. Mater. 11, $103(2012)$

98. J. Chakhalian, J.W. Freeland, A.J. Millis, C. Panagopoulos, J.M. Rondinelli, Rev. Mod. Phys. 86, 1189 (2014)

99. C.A. Jackson, J.Y. Zhang, C.R. Freeze, S. Stemmer, Nat. Commun. 5, 4258 (2014)

100. R. Chen, S.B. Lee, L. Balents, Phys. Rev. B 87, 161119(R) (2013)

101. F. Lechermann, M. Obermeyer, New J. Phys. 17, 043026 (2015)

102. A.C. Komarek, H. Roth, M. Cwik, W.D. Stein, J. Baier, M. Kriener, F. Bourée, T. Lorenz, M. Braden, Phys. Rev. B 75, 224402 (2007)

103. F. Lechermann, arXiv:1603.01031 (2016)

104. L. Boehnke, F. Lechermann, Phys. Rev. B 85, 115128 (2012) 
105. A. Wilhelm, F. Lechermann, H. Hafermann, et al., Phys. Rev. B 91, 155114 (2015)

106. H. Ohta, K. Yoshimura, Z. Hu, Y.Y. Chin, H.J. Lin, H.H. Hsieh, C.T. Chen, L.H. Tjeng, Phys. Rev. Lett. 107, 066404 (2011)

107. L. Boehnke, H. Hafermann, M. Ferrero, F. Lechermann, O. Parcollet, Phys. Rev. B 84, 075145 (2011)

108. N.L. Wang, P. Zheng, D. Wu, Y.C. Ma, T. Xiang, R.Y. Jin, D. Mandrus, Phys. Rev. Lett. 93, 237007 (2004)

109. U. Schollwöck, Ann. Phys. (N.Y.) 326, 96 (2011)

110. J. Braun, R. Rausch, M. Potthoff, J. Minar, H. Ebert, Phys. Rev. B 91, 035119 (2015)

111. H. Aoki, N. Tsuji, M. Eckstein, M. Kollar, T. Oka, P. Werner, Rev. Mod. Phys. 86, $779(2014)$

112. Y. Ge, J. Eisert, New J. Phys. 18, 083026 (2016)

113. P.E. Blöchl, T. Pruschke, M. Potthoff, Phys. Rev. B 88, 205139 (2013) 